\title{
Food-specific IgGs Are Highly Increased in the Sera of Patients with Inflammatory Bowel Disease and Are Clinically Relevant to the Pathogenesis
}

\author{
Nanping Xiao ${ }^{1,2}$, Fenghua Liu ${ }^{3}$, Guangxi Zhou ${ }^{3}$, Mingming Sun ${ }^{3}$, \\ Fengfu $\mathrm{Ai}^{4}$ and Zhanju Liu ${ }^{1,3}$
}

\begin{abstract}
:
Objective Dietary antigens are common luminal antigens in the gastrointestinal tract and have been considered to contribute to the pathogenesis of inflammatory bowel disease (IBD). We analyzed the levels of foodspecific IgGs against a variety of dietary antigens, explored the clinical relevance of food allergy to the pathogenesis of IBD, and investigated whether or not infliximab (IFX) treatment could regulate the immune responses induced by dietary antigens.

Methods A total of 301 IBD patients, including 201 patients with Crohn's disease (CD) and 100 patients with ulcerative colitis (UC), were recruited, and their serum food-specific IgGs against 14 food antigens were detected by a semi-quantitative enzyme linked immunosorbent assay (ELISA). Total serum IgG and IgE levels were measured by immunonephelometry and fluorescent enzyme immunoassay, respectively. Simultaneously, the relevant medical records and clinical data were collected for further analyses.

Results Food-specific IgGs against egg, milk, wheat, corn, rice, tomato, codfish, and soybean antigens were found to be significantly increased in the sera of CD patients compared with UC patients and healthy controls $(\mathrm{p}<0.01)$. The levels of total serum $\operatorname{IgG}$ and $\operatorname{IgE}$ were also significantly higher in CD patients than in healthy controls $(\mathrm{p}<0.01)$. The titers of corn- and tomato-specific IgGs were found to be significantly correlated with total serum IgG in CD patients $(\mathrm{p}<0.05)$, while the titers of egg-, milk-, and wheat-specific IgGs were correlated with total serum IgE $(\mathrm{p}<0.05)$. Interestingly, IFX therapy was able to down-regulate the foodspecific IgG-mediated immune response markedly in active CD patients.

Conclusion Food-specific IgGs against egg, milk, wheat, corn, rice, tomato, codfish, and soybean are highly increased in the sera of CD patients. IFX treatment was able to down-regulate the levels of foodspecific IgGs by suppressing intestinal inflammation and promoting mucosal healing. Therefore, food-specific IgGs may serve as an important approach in the diagnosis and management of food allergy in IBD.
\end{abstract}

Key words: food-specific IgGs, food antigens, total serum IgG, total serum IgE, inflammatory bowel disease

(Intern Med 57: 2787-2798, 2018)

(DOI: 10.2169/internalmedicine.9377-17)

\section{Introduction}

Crohn's disease (CD) and ulcerative colitis (UC) are two major subtypes of inflammatory bowel diseases (IBD), which are chronic relapsing inflammatory diseases of the gastrointestinal tract with increasing prevalence throughout the world. IBD occurs mostly among young people and often leads to a greatly decreased quality of life, characterized by increased stool frequency, purulent and bloody stools, abdominal pain, and loss of weight (1-4). Increasing evidence has demonstrated that IBD results from the dys-

\footnotetext{
${ }^{1}$ Department of Gastroenterology, The Shanghai Tenth Clinical Medicine College of Nanjing Medical University, China, ${ }^{2}$ Department of Gastroenterology, Sichuan Guangyuan First People's Hospital, China, ${ }^{3}$ Department of Gastroenterology, The Shanghai Tenth People's Hospital of Tongji University, China and ${ }^{4}$ Department of Internal Medicine, The Second People's Hospital of Linchuan district, China Received: April 20, 2017; Accepted: October 22, 2017; Advance Publication by J-STAGE: May 18, 2018 Correspondence to Dr. Zhanju Liu, liuzhanju88@126.com
} 
regulation of the mucosal immune response toward commensal bacterial flora along with genetic and environmental factors (5-7).

Efforts have been made to explore the link between IBD and atopy. Bernstein et al. (8) analyzed over 8,000 IBD patients and found that asthma was the most common comorbidity in $\mathrm{CD}$ patients. The increased prevalence of atopic diseases, including eczema, asthma, and allergic rhinitis, was also reported in IBD (9). Specially, patients with IBD exhibit features resembling those of asthma, such as increased eosinophil counts in induced sputum and increased airway hyperresponsiveness, suggesting a close relationship between IBD and atopy and a shared tendency for mucosal inflammation in the lungs and gut $(10,11)$. Actually, in addition to neutrophils and lymphocytes, previous studies on the histological features of IBD have shown the infiltration of eosinophils in intestinal lesions (12). Furthermore, increased mast cell numbers and intestinal (tissue or luminal) levels of mast cell mediators, as well as elevated mast cell degranulation, have also been detected in IBD patients, especially CD patients (13).

To date, studies concerning food intolerance in IBD have largely focused on classic food allergies based on IgEmediated antibody responses $(14,15)$. The levels of total or food-specific IgEs have been observed to be increased in the sera of IBD patients $(14,16)$, and IgE-mediated food allergies are more frequent in IBD patients than in those without IBDs $(17,18)$. Nevertheless, reactions mediated by foodspecific IgGs, featuring a more delayed response following exposure to a particular antigen, are also expected to contribute to adverse reactions in IBD (15), and food-specific IgGs help physicians identify the candidate food for elimination in IBD patients (19). Furthermore, IgG-mediated adverse reactions have also been reported to be involved in some cases of food hypersensitivity $(20,21)$.

Abdominal pain, vomiting and diarrhea are general symptoms of gastrointestinal food allergy resulting from immune responses. IBD and food allergy may become associated comorbidities through their pathophysiological progress. In IBD patients, because of gastrointestinal inflammation and the subsequent treatment with antibiotics, bacterial dysbiosis often occurs, breaking the immunologic tolerance to food antigens, which further facilitates the incidence of food allergy (22). Moreover, allergies to certain foodstuffs enhance the risk of IBD in genetically susceptible individuals. However, the mechanisms underlying the observed association between IBD and food allergy have yet to be defined.

With this background, the present study examined the immune responses to dietary antigens in IBD patients and explored the clinical relevance of food allergy to the pathogenesis of IBD. We compared the levels of food-specific IgGs against 14 food antigens (rice, egg, mushroom, milk, pork, chicken, beef, crab, codfish, corn, soybean, tomato, shrimp, and wheat) between IBD patients and healthy controls. The correlation between food-specific IgGs and total serum $\operatorname{IgG}$ and $\operatorname{IgE}$ levels was also investigated. Finally, we examined whether or not infliximab (IFX) treatment was able to regulate the immune responses induced by food antigens.

\section{Materials and Methods}

\section{Patients}

This study recruited a total of 301 patients with IBD (CD, $\mathrm{n}=201$; UC, $\mathrm{n}=100$ ) and 178 healthy controls from June 2013 to January 2016 at the Department of Gastroenterology, the Shanghai Tenth People's Hospital of Tongji University (Shanghai, China). The diagnoses of CD and UC were based on clinical, radiological, endoscopic, and histological criteria. The disease phenotypes of CD patients (age at the onset, disease location, and behavior) were determined according to the Montreal classification (23). The disease location of UC patients was determined from the endoscopic findings. The disease activity was scored by the Crohn's disease activity index (CDAI) in CD patients (24) and the Clinical Activity Index (CAI) in UC patients (25), and the severity of intestinal mucosal lesions was graded by the simple endoscopic score for CD (SES-CD) (26). According to the consensus on the diagnosis of IBD drawn up by European Crohn's and Colitis Organization (ECCO) $(27,28)$, each patient met the diagnostic criteria for $\mathrm{CD}$ or $\mathrm{UC}$, and serum food-specific IgGs were tested during the hospital visit. Another 178 healthy volunteers were randomly chosen to represent the general population as healthy controls, whose checkups were finished in our hospital as a routine item. Written informed consent was obtained from each participant before experiments. The study was approved by the Ethics Committee of the Shanghai Tenth People's Hospital. All studies involving human subjects conformed to the provisions of the latest revision of World Medical Association's Declaration of Helsinki.

\section{Data collection}

The medical records and clinical data of all selected IBD patients were comprehensively reviewed, and the general demographic data were summarized with details. The clinical data of IBD patients regarding their gender, age, body mass index (BMI), duration, classification, treatment (e.g., 5-aminosalicylate, corticosteroids, azathioprine, and IFX), CDAI, SES-CD, and laboratory parameters, such as Creactive protein (CRP), erythrocyte sedimentation rate (ESR), hemoglobin ( $\mathrm{Hb}$ ), and albumin (Alb), were collected.

\section{An enzyme-linked immunosorbent assay for food- specific IgG antibodies against 14 common food- derived antigens}

An enzyme-linked immunosorbent assay (ELISA) for the semi-quantitative analysis of serum food-specific IgGs against 14 food-derived antigens (i.e., rice, egg, mushroom, milk, pork, chicken, beef, crab, codfish, corn, soybean, tomato, shrimp, and wheat) was performed using a detection 
kit (Biomerica, Newport Beach, USA) according to the operation manual. Briefly, the samples were first diluted (1: 100) and loaded into the microwells which were precoated with 14 food-derived antigens together with blanks and positive controls. The loaded microwells were incubated at room temperature for 60 minutes, and $100 \mu \mathrm{L}$ of food-specific IgG-HRP conjugate was then added into each well. Thereafter, the plates were incubated for another 30 minutes at room temperature, and $100 \mu \mathrm{L}$ of working substrate mixture were added to each well. The reaction was stopped using 50 $\mu \mathrm{L}$ of $1 \mathrm{~N}$ sulphuric acid per well. Finally, the optical densities were read using an ELISA plate reader (BioTek, Winooski, USA) at $450 \mathrm{~nm}$. A level of food-specific IgGs $<50$ $\mathrm{U} / \mathrm{mL}$ was considered negative (marked as “-”), while values of $50-100,100-200$, and $\geq 200 \mathrm{U} / \mathrm{mL}$ were referred to as grade +1 (mild sensitivity), grade +2 (moderate sensitivity), and grade +3 (high sensitivity), respectively.

\section{Total serum IgG \& IgE assay}

The total serum IgG level was measured by immunonephelometry on a Beckman Image Immunochemistry System using standard laboratory techniques (Beckman Coulter, Brea, USA; reference range for healthy people: total serum IgG: 7-16 g/L). The total serum IgE level was determined with a fluorescent enzyme immunoassay by ImmunoCAP (Phadia; Uppsala, Sweden) and expressed as kU/ L.

\section{Flow cytometry}

Cells were fixed by $2 \%$ paraformaldehyde for 1 hour. After washing with phosphate buffered saline, cells were incubated with PE-conjugated anti-CD20 (BD PharMingen; San Diego, USA). The cells were analyzed by a flow cytometry (BD FACSCanto II, BD Biosciences; San Diego, USA).

\section{Detection of food-specific IgGs before and after IFX treatment}

Thirty-one patients were all naïve to biological agent therapy and received anti-TNF mAb (5 mg/kg IFX; Cilag, Schaffhausen, Switzerland) treatment at weeks 0, 2, and 6. Infusions were delivered over a 2 -h period. After the first session of IFX therapy, the patients were monitored weekly during follow-up, and serum samples were collected at weeks 0 and 10 after the first infusion and analyzed for food-specific IgGs according to the method described previously.

\section{Data analyses}

Continuous numerical variables are expressed as the mean \pm standard deviation (SD). Differences in the levels of foodspecific IgGs against 14 dietary antigens between groups were analyzed by a one-way analysis of variance (ANOVA), with Tamhane's T2 test used in cases of non-homogeneity of variances. Pearson's correlation was used to evaluate the correlation between food-specific IgGs against dietary antigens and total serum IgG, total serum IgE, and CDAI. The chi-squared test and Fisher's exact test were used to test for an association between positive food-specific $\operatorname{IgG}$ distribution and age, sex, disease location, and disease behavior. Parameters, including food-specific IgGs against 14 dietary antigens, CRP, ESR, Hb, Alb, and SES-CD, were compared before and 10 weeks after IFX induction using Student's $t$ test. Similar immune responses induced by 14 dietary antigens among IBD patients and healthy controls were clustered by a hierarchical cluster analysis using the Ward method and the squared Euclidean distances. A discriminant analysis was used to distinguish between the groups (CD, UC, healthy controls), and multinomial logistic regression was used to fit models predicting group membership.

All statistical analyses were performed using the PASW Statistics software program (version 18.0; SPSS, Chicago, USA). Graphs were generated in the Graphpad Prism software program (version 3.02; GraphPad Software, San Diego, USA). A p value of less than 0.05 (2-sided significance testing) was regarded as statistically significant in all analyses.

\section{Results}

\section{Demographic data of all subjects}

The demographics and clinical characteristics of IBD patients and healthy controls (HCs) are shown in Table 1. A total of 301 IBD patients, including $201 \mathrm{CD}$ (130 males and 71 females) and 100 UC (62 males and 38 females) patients, as well as $178 \mathrm{HCs}$ (115 males and 63 females) were recruited for our study. In the $\mathrm{CD}$ group, the mean diagnostic age was $35.14 \pm 14.21$ years. The average disease duration was $44.41 \pm 53.90$ months, and the mean BMI was 19.94 \pm 3.38. A total of $52.24 \%(105 / 201)$ of patients had ileocolonic lesions, and $50.25 \%(101 / 201)$ had received IFX treatment. However, the mean diagnostic age was $45.87 \pm 17.20$ years in the UC group. The average disease duration was $34.56 \pm 36.50$ months, and the mean BMI was 21.65 \pm 3.40 . The proportions of proctitis, left-sided, and extensive type were $10 \%(10 / 100), 27 \%(27 / 100)$, and $63 \%$ (63/100), respectively. A total of $58 \%(58 / 100)$ of the patients had received treatment with 5-ASA and corticosteroid drugs. The majority of CD patients $(68.66 \%, 138 / 201)$ were in the active stage, while more than half of UC patients (57\%, 57/ 100) were in remission or had a mild disease activity (Table 1).

\section{A comparison of food-specific IgGs against $14 \mathrm{com}$ - mon food-derived antigens between IBD patients and HCs}

The mean levels of food-specific IgGs for each of the 3 groups against 14 food-derived antigens are shown in Table 2. CD patients had significantly higher levels of foodspecific IgGs than the other 2 groups against corn $(\mathrm{p}<0.01)$, codfish $(\mathrm{p}<0.01)$, soybean $(\mathrm{p}<0.01)$, milk $(\mathrm{p}<0.01)$, rice $(\mathrm{p}<$ $0.01)$, wheat $(\mathrm{p}<0.01)$, egg $(\mathrm{p}<0.01)$, and tomato antigens $(\mathrm{p}$ $<0.01)$. No significant differences were observed in beef- ( $p$ 
Table 1. Baseline Demographic and Clinical Characteristics of IBD Patients.

\begin{tabular}{|c|c|c|c|}
\hline Variables & $\mathrm{CD}(\mathrm{n}=201)$ & $\mathrm{UC}(\mathrm{n}=100)$ & $\mathrm{HC}(\mathrm{n}=178)$ \\
\hline Females $(\%)$ & $71(35.32 \%)$ & $38(38 \%)$ & $63(35.39 \%)$ \\
\hline \multirow[t]{4}{*}{ Age at diagnosis (years) } & $35.14 \pm 14.21(13,74)$ & $45.87 \pm 17.20(17,83)$ & $47.36 \pm 10.78(24,80)$ \\
\hline & A1 ( $\leq 16$ years): $14(6.97 \%)$ & & \\
\hline & A2 (17-40 years): $122(60.70 \%)$ & & \\
\hline & A3>40 years: $65(32.34 \%)$ & & \\
\hline BMI $\left(\mathrm{kg} / \mathrm{m}^{2}\right)$ & $19.94 \pm 3.38(13.02,29.39)$ & $21.65 \pm 3.40(13.22,28.28)$ & \\
\hline Duration (months) & $44.41 \pm 53.90(0.25,360)$ & $34.56 \pm 36.50(0.5,168)$ & \\
\hline \multirow[t]{6}{*}{ Location of disease } & L1-Terminal ileum: 43 (21.39\%) & E1-Proctitis: 10 (10\%) & \\
\hline & L2-Colon: 49 (24.38\%) & E2-Left-sided colitis: 27 (27\%) & \\
\hline & L3-Ileocolon: 105 (52.24\%) & E3-Extensive/pancolitis: 63 (63\%) & \\
\hline & L4-Upper gastrointenstina: 8 (3.98\%) & & \\
\hline & L2+L4: $2(1 \%)$ & & \\
\hline & L3+L4: $2(1 \%)$ & & \\
\hline \multirow{4}{*}{ Disease behavior } & B1-Non stricturing, non penetrating : $91(45.27 \%)$ & & \\
\hline & B2-Stricturing: $83(41.29 \%)$ & & \\
\hline & B3-Penetrating: 27 (13.43\%) & & \\
\hline & P-Perianal disease: $15(7.46 \%)$ & & \\
\hline \multirow[t]{4}{*}{ Disease activity } & Remission: 63 (31.34\%) & Remission: 18 (18\%) & \\
\hline & Active: $138(68.66 \%)$ & Mild: $39(39 \%)$ & \\
\hline & & Moderate: 25 (25\%) & \\
\hline & & Severe: $18(18 \%)$ & \\
\hline \multirow[t]{2}{*}{ Incipient/Recurrence } & & Incipient: $38(38 \%)$ & \\
\hline & & Recurrence: $62(62 \%)$ & \\
\hline \multicolumn{4}{|l|}{ Treatment } \\
\hline 5-ASA & $142(70.65 \%)$ & $97(97 \%)$ & \\
\hline Azathioprine & $76(37.62 \%)$ & $17(17 \%)$ & \\
\hline Corticosteroids & $82(40.59 \%)$ & $75(75 \%)$ & \\
\hline IFX & $101(50.25 \%)$ & $5(5 \%)$ & \\
\hline Enteral nutrition & $29(14.36 \%)$ & $6(6 \%)$ & \\
\hline Surgical intervention & $58(28.71 \%)$ & $1(1 \%)$ & \\
\hline
\end{tabular}

CD: Crohn's disease, UC: ulcerative colitis, HC: healthy controls, BMI: body mass index, 5-ASA: 5-aminosalicylate, IFX: infliximab

Table 2. Levels of Food-specific IgGs against 14 Food Antigens between IBD Patients and Healthy Controls.

\begin{tabular}{|c|c|c|c|c|c|c|c|}
\hline & \multicolumn{2}{|c|}{$\mathrm{CD}(\mathrm{n}=201)$} & \multicolumn{2}{|c|}{$\mathrm{UC}(\mathrm{n}=100)$} & \multicolumn{2}{|c|}{ Control $(\mathrm{n}=178)$} & \multirow[b]{2}{*}{$\mathrm{p}$ value } \\
\hline & Mean & $\begin{array}{l}\text { Standard error } \\
\text { of mean }\end{array}$ & Mean & $\begin{array}{l}\text { Standard error } \\
\text { of mean }\end{array}$ & Mean & $\begin{array}{l}\text { Standard error } \\
\text { of mean }\end{array}$ & \\
\hline Beef & 0.83 & 4.59 & 0.15 & 0.77 & 0.93 & 4.89 & 0.30 \\
\hline Chicken & 3.91 & 22.90 & 0.61 & 2.87 & 0.89 & 3.34 & 0.08 \\
\hline Codfish & $17.23 * * \# \#$ & 53.73 & 3.95 & 11.75 & 4.17 & 10.47 & 0.00 \\
\hline Corn & $83.28 * * \# \#$ & 114.79 & 7.55 & 9.90 & 10.67 & 14.55 & 0.00 \\
\hline Crab & 11.42\# & 19.01 & 7.04 & 14.21 & 8.48 & 17.51 & 0.09 \\
\hline Egg & $150.06^{* * \# \#}$ & 199.89 & 54.21 & 109.42 & 36.18 & 61.38 & 0.00 \\
\hline Mushroom & 16.45 & 38.48 & 10.30 & 12.47 & 11.46 & 15.03 & 0.10 \\
\hline Milk & $53.62 * * \# \#$ & 115.18 & 19.13 & 50.55 & 8.69 & 22.78 & 0.00 \\
\hline Pork & 9.65 & 51.53 & 3.33 & 11.80 & 3.81 & 10.60 & 0.17 \\
\hline Rice & $73.52 * * \# \#$ & 94.89 & 3.00 & 6.79 & 5.94 & 10.93 & 0.00 \\
\hline Shrimp & 8.99 & 26.47 & 4.11 & 9.68 & 10.09 & 18.81 & 0.07 \\
\hline Soybean & $53.76^{* * \# \#}$ & 92.96 & 6.39 & 21.58 & 4.78 & 14.43 & 0.00 \\
\hline Tomato & $79.62 * * \# \#$ & 120.52 & 10.77 & 27.82 & 12.34 & 21.88 & 0.00 \\
\hline Wheat & $33.94 * * \# \#$ & 64.30 & 7.55 & 10.69 & 10.75 & 21.86 & 0.00 \\
\hline
\end{tabular}

**p $<0.01, \mathrm{CD}$ patients vs healthy controls; \#p $<0.05$, \#\# $<0.01 \mathrm{CD}$ patients vs UC patients. CD: Crohn's disease, UC: ulcerative colitis 


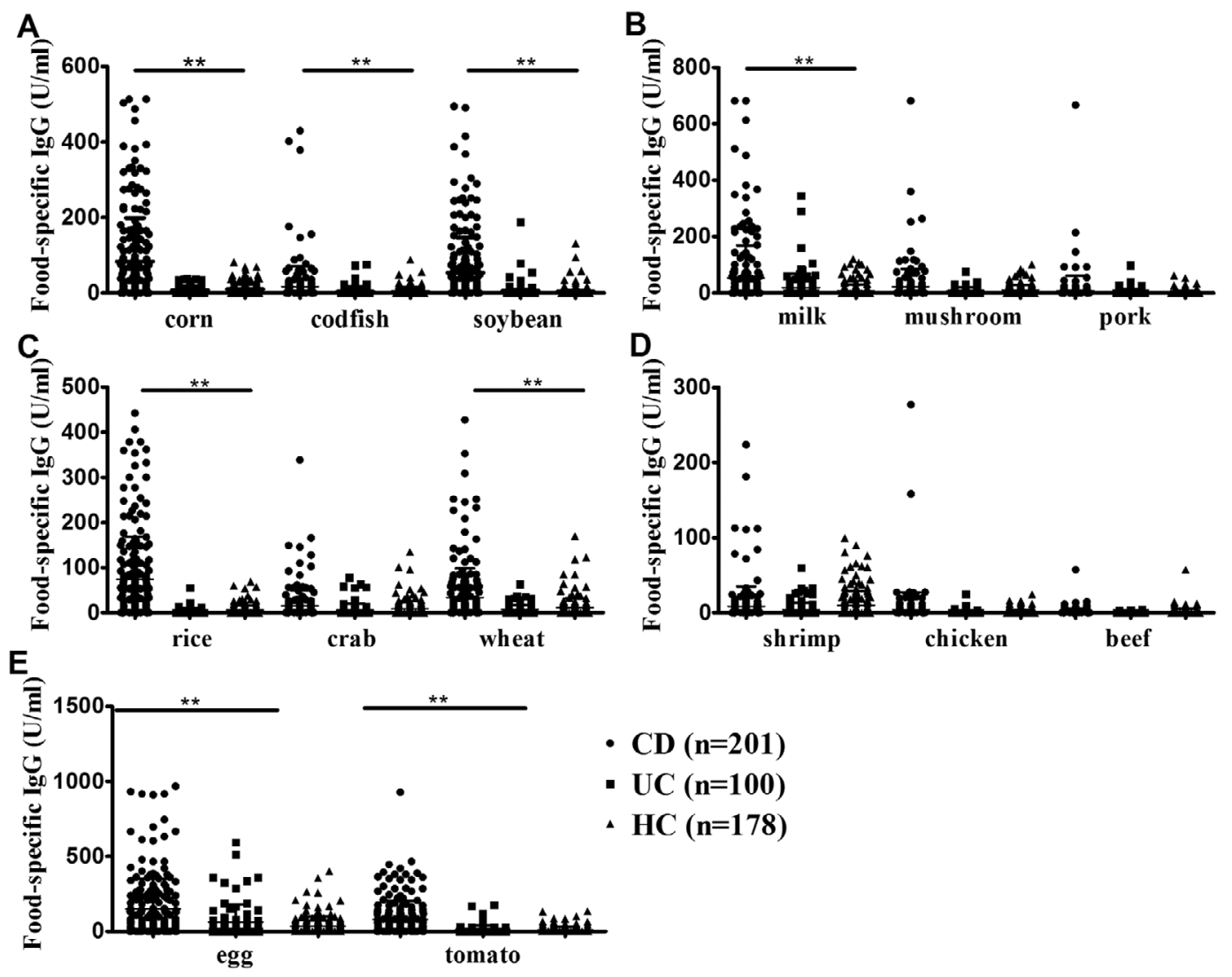

Figure 1. The levels of food-specific IgGs against 8 common daily foods increased markedly in CD patients. A comparison of food-specific IgGs against corn, codfish, and soybean (A); milk, mushroom, and pork (B); rice, crab, and wheat $(C)$; shrimp, chicken, and beef (D); and egg and tomato (E) between CD patients, UC patients, and HCs. $* *$ p<0.01. CD: Crohn's disease, UC: ulcerative colitis, HCs: healthy controls

$=0.3)$, shrimp- $(\mathrm{p}=0.07)$, crab- $(\mathrm{p}=0.09)$, chicken- $(\mathrm{p}=0.08)$ pork- $(\mathrm{p}=0.17)$, or mushroom- $(\mathrm{p}=0.10)$ specific IgGs compared with HCs. However, no significant differences in the food-specific IgGs against 14 dietary antigens were observed between UC patients and HCs (Table 2 and Fig. 1).

A discriminant analysis showed that patients with $\mathrm{CD}$ could be differentiated by their food-specific IgGs against 14 food antigens, and in the 2-dimensional plot, the clustering between patients with $\mathrm{CD}$ and HCs was readily apparent; however, no obvious separation was observed between the UC group and HCs (Fig. 2). Statistical classification based on multinomial logistic regression demonstrated the predictive value to be $74.6 \%$ for the CD group, $88.2 \%$ for $\mathrm{HCs}$, and $11 \%$ for the UC group, respectively (Supplementary material 1). A cluster analysis suggested that there might be shared allergenic relationships when food-specific IgGs against 14 food antigens from patients with IBD and HCs were considered together. For instance, there appeared to be associations between corn and rice, between mushroom and pork, and between beef, chicken, shrimp, and crab (Fig. 3).
Correlation of food-specific IgGs with the total serum levels of IgG, IgE, and CDAl in patients with $C D$

A Pearson analysis was performed between food-specific IgGs (against corn, codfish, soybean, milk, rice, wheat, egg, and tomato antigens) and total serum $\operatorname{IgG}$ and $\operatorname{IgE}$ levels in $\mathrm{CD}$ patients. The average levels of total serum $\mathrm{IgG}$ in patients with $\mathrm{CD}$ and UC and HCs were 13.94 $\pm 3.98,12.39 \pm$ 4.30, and $11.42 \pm 3.30 \mathrm{~g} / \mathrm{L}$, respectively. CD patients had markedly higher levels of total serum $\operatorname{IgG}$ than HCs $(\mathrm{p}<$ 0.001), whereas UC patients did not (Fig. 4A). CD patients showed significantly positive correlations between foodspecific IgGs against corn $(\mathrm{r}=0.23, \mathrm{p}<0.01)$, tomato $(\mathrm{r}=$ $0.173, \mathrm{p}<0.05)$, and the levels of total serum IgG. However, no significant correlations of food-specific IgGs against codfish $(\mathrm{p}=0.288)$, egg $(\mathrm{p}=0.397)$, milk $(\mathrm{p}=0.391)$, wheat $(\mathrm{p}=$ $0.097)$, rice $(\mathrm{p}=0.085)$, or soybean $(\mathrm{p}=0.910)$ with total serum IgG were found (Table 3).

The average levels of total serum IgE from subjects with $\mathrm{CD}$ and UC and HCs were 110.72 \pm 224.23 , 48.61 \pm 90.55 , and $29.45 \pm 25.09 \mathrm{kU} / \mathrm{L}$, respectively. The average levels of total serum $\operatorname{IgE}$ in $\mathrm{CD}$ patients differed significantly from those in HCs $(\mathrm{p}<0.01)$, while there was no marked difference between UC patients and HCs (Fig. 4B). CD patients 


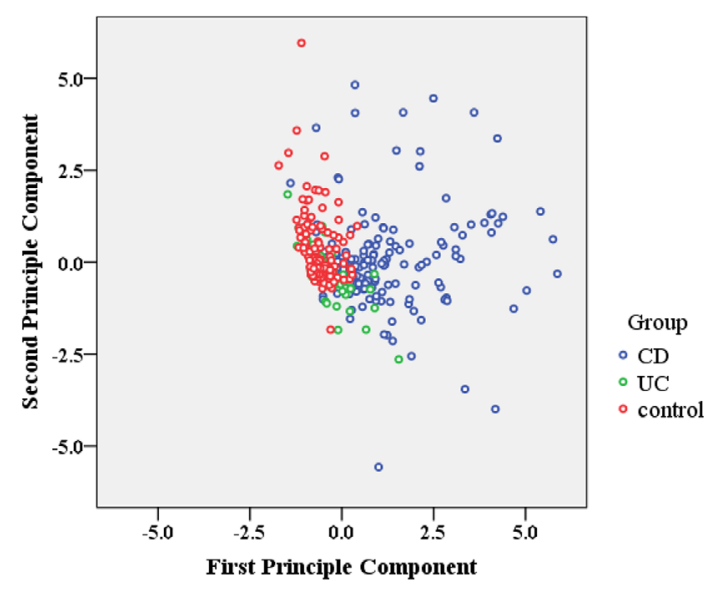

Figure 2. Classification of IBD patients and HCs. A linear discriminant analysis revealed a weighted linear combination of the 14 "independent variables" (all food-specific IgGs against 14 food antigens) where the three groups $(\mathrm{CD}, \mathrm{UC}$ and HCs) differed as much as possible. IBD: inflammatory bowel disease

showed significantly positive correlations of food-specific IgGs against egg $(r=0.219, \mathrm{p}<0.05)$, milk $(\mathrm{r}=0.204, \mathrm{p}<0.05)$, and wheat $(\mathrm{r}=0.274, \mathrm{p}<0.01)$ with the levels of total serum IgE. Nevertheless, no significant correlations of foodspecific IgGs against codfish $(\mathrm{p}=0.223)$, corn $(\mathrm{p}=0.575)$, rice $(\mathrm{p}=0.878)$, tomato $(\mathrm{p}=0.873)$, or soybean $(\mathrm{p}=0.742)$ with total serum IgE were seen (Table 3 ).

In addition, a Pearson analysis showed no correlation between food-specific IgGs against eight food antigens (corn, codfish, soybean, milk, rice, wheat, egg, and tomato antigens) and CDAI (Table 3).

The relationship between food-specific IgGs and age, sex, disease location, and disease behavior

No significant correlations were observed between positive food-specific IgG distribution and sex $(p=0.685)$, disease location $(p=0.605)$, or disease behavior $(p=0.758)$ in $\mathrm{CD}$ patients. However, correlations between age and positive food-specific $\mathrm{IgG}$ distribution were observed $(\mathrm{p}<0.05)$ (Supplementary material 2 ).

Effects of IFX treatment on the levels of food specific-IgGs against 14 common food antigens

Our previous study demonstrated that IFX treatment was able to improve the intestinal barrier (29). Therefore, we examined whether or not this kind of therapy could regulate the levels of food-specific IgGs in the sera from CD patients. To this end, we chose 31 patients in whom foodspecific IgGs against 14 food antigens had been measured before and 10 weeks after IFX induction, as described previously (29). Among those patients, 19 showed a reduced number of positive food allergens and a decreased degree of food allergen sensitivity simultaneously, 10 showed a reduced degree of food allergen sensitivity with an unchanged number of positive food allergens, and only 2 showed an in-

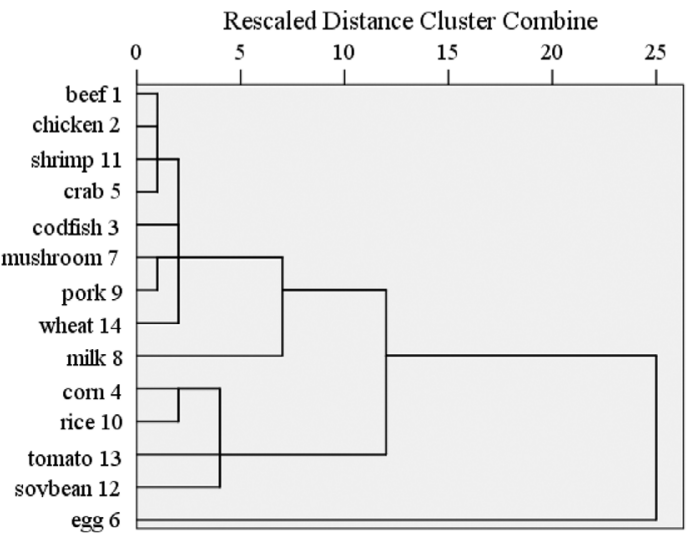

Figure 3. The presence of cross-reactive antigen epitopes between food antigens. A dendrogram of all food-specific IgG data. The dendrogram was developed using the Ward method of clustering to show food antigens that elicited similar immune responses in IBD patients and HCs.

creased food allergen sensitivity (Table 4). Similarly, as shown in Supplementary material 3, IFX treatment reduced the levels of food-specific IgGs in these CD patients, although it did not reach statistical difference, except for eggspecific IgG $(\mathrm{p}<0.01)$. The levels of serum CRP $(\mathrm{p}<0.01)$, ESR $(p<0.01)$, and SES-CD $(p<0.01)$ were found to be significantly decreased, while the levels of $\mathrm{Hb}(\mathrm{p}<0.01)$ and Alb $(\mathrm{p}<0.01)$ were markedly increased after IFX treatment (Fig. 5). We also noted that the levels of total serum IgE and percentages of $\mathrm{CD} 20^{+} \mathrm{B}$ cells in peripheral blood were not markedly different after IFX treatment from before (Supplementary material 4). Therefore, these results suggest that IFX may help reduce the potential food allergic response by suppressing intestinal inflammation and promoting mucosal healing.

\section{Discussion}

The gastrointestinal tract, which is the largest immunologic organ in the body, is constantly exposed to an enormous array of exogenous antigens, including commensal bacteria and ingested food proteins. The intestine has the unenviable task of identifying and responding to constant environmental stimuli that can be both dangerous and beneficial. Therefore, failure to induce tolerance to food antigens is thought to result in food allergies, which is the most prevalent food-induced pathology (30). IBD results from complex interactions involving dysregulated immune responses to the microbiota in the gut lumen. Studies on gut mucosal antigen behavior have shown that in vivo and in vitro data from $\mathrm{CD}$ patients both exhibit sensitization in response to specific food antigens (31). The ingestion of different categories of food, e.g., yeast, wheat, corn, and vegetables, sometimes contributes to the worsening of clinical symptoms in CD patients. Therefore, dietary elimination is recommended and may be beneficial (19), suggesting the di- 


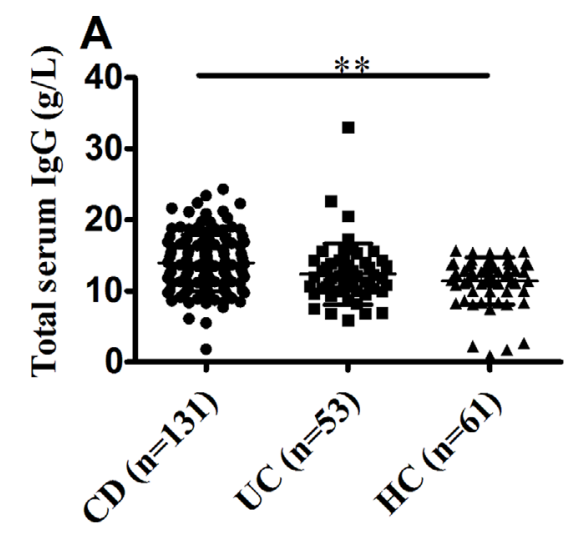

B

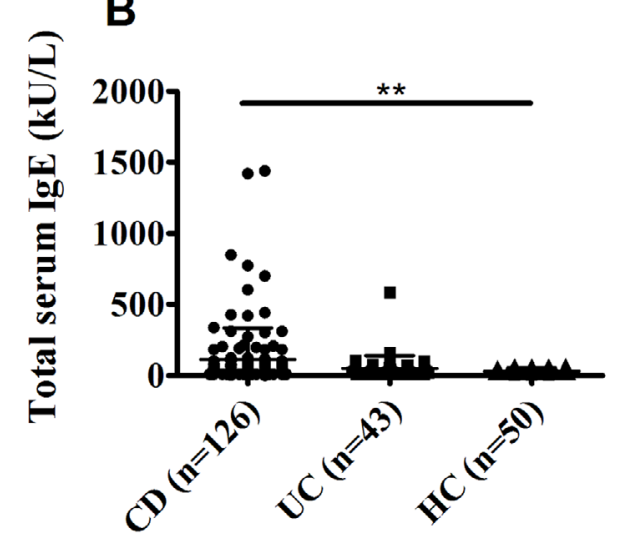

Figure 4. A comparison of the total serum IgG and IgE levels between CD patients, UC patients, and HCs. The levels of serum IgG and IgE were determined using the method indicated. A: Total serum IgG levels in CD patients $(n=131)$, UC patients $(\mathrm{n}=53)$, and HCs $(\mathrm{n}=61)$. B: Total serum IgE levels in CD patients $(\mathrm{n}=126)$, UC patients $(\mathrm{n}=43)$, and HCs $(\mathrm{n}=50)$. $* * \mathbf{p}<0.01$ CD.

rect association of the food intake with the pathophysiology of CD. However, thus far, immunological evidence for dietary allergies in $\mathrm{CD}$ has remained elusive.

In the current study, statistical analyses demonstrated an obvious increase in the levels of food-specific IgGs in Chinese $\mathrm{CD}$ patients compared with $\mathrm{HCs}$ against codfish, egg, milk, wheat, rice, corn, tomato, and soybean antigens, consistent with previous studies (32-34). The selection of the 14 food-derived antigens was based on three immediate concerns: first, the 14 food-derived antigens have been reported to be associated with IBD (35); second, the 14 food-derived antigens were the most common daily foods in China; third, all 14 food-derived antigens can be determined in one detection batch using the Allerquant Food Allergy Screening ELISA Kit (Biomerica). In this way, the results may be more accurate than in other studies, as all evaluations were performed using the same detection kit. However, considering the subjectivity of the present investigation, we will include more food-associated antigens to encompass more food allergenic symptoms and improve the efficiency of allergen selection in future studies. High levels of foodspecific IgGs against grains and vegetables in $\mathrm{CD}$ patients and against soybean, corn, and wheat in IL-10-deficient mice with colitis have been observed (32). The selective elevation of soybean-specific IgG4 was also reported in CD but not in UC patients (33). In another study, Lerner et al. reported that patients with $\mathrm{CD}$ had higher IgGs against BSA and $\beta$-lactoglobulin A and B than HCs (34). Under other atopic conditions, increased IgGs against food antigens have also been reported. Patients with eczema and asthma caused by milk intolerance have shown raised levels of IgG4 (36). Similarly, Awazuhara et al. (20) demonstrated strongly reactive IgG4 in patients with atopic dermatitis and/or bronchial asthma (caused by soybean hypersensitivity). Food-allergic patients (e.g., peanut allergy and milk allergy) also showed high levels of allergen-specific IgGs $(37,38)$. Thus, elevated food-specific IgGs may play an important etiologic role in the pathogenesis of $\mathrm{CD}$ patients, suggesting that $\mathrm{CD}$ is closely correlated with atopy diseases.

The extent of antibody responses to dietary antigens is the result of many factors. Regarding protein-rich products, such as cow's milk, codfish, and egg, the antigenicity of a specific protein is of great importance. It was reported that, in cow's milk, BSA, BLG, and bovine gammaglobulin are the most antigenic proteins despite their low concentrations. Specially, caseomorphin, a specific component of casein, is found to evoke chemotactic responses, which may be involved in the perpetuation of inflammatory process (34). Jowett et al. (39) have suggested that undigested sulfur proteins may have multiple functions within the colon; for example, their metabolism provides energy for intestinal bacteria, but their final metabolites (e.g., hydrogen sulfide) may exert toxic effects on the intestines, which may result in intestinal epithelial dysfunction, thereby contributing to the occurrence of IBD. Foodstuffs such as rice, wheat, corn, soybean, and tomato are traditional products and some of the most commonly used ingredients in China. As such, most Chinese people are frequently exposed to these intestinal antigens. In the study by Bentz, it was found that the elimination of allergen foods from the diet resulted in a decrease in the IgG levels (19), suggesting a close relationship between the levels of food-specific IgGs and the amount of antigen intake. In our study, high antibody titers were observed for codfish, egg, milk, wheat, rice, corn, tomato, and soybeans. Relevant mechanisms may include differences in the intrinsic antigenicity of various foods, the impact of protein structure modifications during digestive processes, or the disturbance of the process concerning antigen load presented to the immune cells associated with the differential permeability of the gut mucosa to different foods. Therefore, different foods may lead to differential modification of the gut immune responses. Of note, no marked changes were observed in the IgG titers against beef, shrimp, crab, chicken, pork, or mushroom between $\mathrm{CD}$ and HCs. This may be related to the fact that $\mathrm{CD}$ patients subconsciously avoid the triggering foods (e.g., beef, shrimp, crab, chicken, pork, and mushroom) to alleviate the antibody response. The $\mathrm{IgG}$ antibody response to different food groups can be a 
Table 3. Correlation of Food-specific IgG Antibodies with Total Serum IgG, IgE and CDAI in Patients with CD.

\begin{tabular}{|c|c|c|c|c|c|c|}
\hline \multirow{2}{*}{ Food-specific IgGs } & \multicolumn{2}{|c|}{ Total serum $\operatorname{IgG}(\mathrm{n}=131)$} & \multicolumn{2}{|c|}{ Total serum $\operatorname{IgE}(n=126)$} & \multicolumn{2}{|c|}{ CDAI $(n=201)$} \\
\hline & $\mathrm{r}$ & $\mathrm{p}$ value & $\mathrm{r}$ & $\mathrm{p}$ value & $\mathrm{r}$ & $\mathrm{p}$ value \\
\hline Codfish & 0.094 & 0.288 & 0.107 & 0.223 & 0.023 & 0.749 \\
\hline Egg & 0.075 & 0.397 & $0.219 *$ & 0.014 & 0.124 & 0.081 \\
\hline Milk & 0.076 & 0.391 & $0.204 *$ & 0.022 & 0.038 & 0.589 \\
\hline Rice & 0.151 & 0.085 & 0.014 & 0.878 & -0.057 & 0.423 \\
\hline Wheat & 0.148 & 0.097 & $0.274 * *$ & 0.002 & -0.024 & 0.738 \\
\hline Corn & $0.23 * *$ & 0.008 & 0.05 & 0.575 & -0.041 & 0.561 \\
\hline Tomato & $0.173 *$ & 0.049 & 0.014 & 0.873 & -0.075 & 0.291 \\
\hline Soybean & 0.01 & 0.91 & 0.03 & 0.742 & -0.008 & 0.912 \\
\hline
\end{tabular}

${ }^{*} \mathrm{p}<0.05,{ }^{* *} \mathrm{p}<0.01$. CD: Crohn's disease, CDAI: Crohn's disease activity index

very complicated process.

Patients with active $\mathrm{CD}$, especially pediatric patients, reportedly have increased intestinal permeability (40). A breached barrier together with active and persistent inflammation enhances the response of the mucosal innate and acquired immune systems to luminal antigens, such as food antigens. Under such circumstances, the levels of foodspecific IgGs may increase. In addition, the fact that lymphoid aggregates are more frequently found in the small intestine than in the colon would result in increased IgG production against luminal antigens in CD patients, whereas in UC patients, colonic inflammation is usually restricted to the mucosa.

Statistical classification based on the discriminant analysis and multinomial logistic regression showed that patients with $\mathrm{CD}$ could be readily distinguished from HCs, suggesting that food-specific IgGs may serve as an aid in the diagnosis of $\mathrm{CD}$. According to the cluster analysis, cross-reactive antigen epitopes between food antigens might exist, but the clinical significance of such cross-reactivity remains to be determined. To obtain accurate allergenic sources regarding individual foods for provoking specific clinical allergies, a thorough analysis of data from carefully obtained clinical histories and the application of double-blind, placebocontrolled food challenges is warranted.

The general increase in the levels of total serum IgGs in $\mathrm{CD}$ patients may reflect the generation of a more robust immune response and may also account for the increased levels of food-specific IgGs against food antigen epitopes. Mast cells express Fc receptors, including $\operatorname{IgE}$ and $\mathrm{IgG}$ receptors, and therefore respond to both $\operatorname{IgE}$ and $\operatorname{IgG}$ (41). Furthermore, serum IgGs have been proven to be involved in allergen- $\operatorname{IgE}$ complexes, which result in binding to $\mathrm{B}$ cells via $\mathrm{CR} 2$ and CD23 and thereby influence the allergic response (42). It has been shown that corn- and tomatospecific IgGs correlate with the total serum IgG level, suggesting the exposure to such food antigens or otherwise indicating the presence of non-specific IgGs cross-reacting with shared epitopes. Accordingly, food-specific IgGs are not an immediate factor functioning in the inhibition in hypersensitivity reaction caused by food intolerance, instead serving as a mediation element.

The function of $\mathrm{IgE}$ is not conclusively defined in gastrointestinal food allergy. Analyses of small bowel aspirates of food-allergic adults, feces of allergic children, and intestinal washings of children with atopic eczema have revealed elevated $\mathrm{IgE}$ levels (43-45). High levels of $\mathrm{IgE}$ are readily produced under normal circumstances in certain rat strains, whereas higher levels of IgE are found to be increased following primary immunization compared with low-responder strains (46). Furthermore, Baird et al. (47) found that the levels of $\operatorname{IgE}$ measured by passive cutaneous anaphylaxis in egg albumin-sensitized rats were proportional to the magnitude of antigen-induced physiological changes. Evidence has shown that patients with $\mathrm{CD}$ had higher levels of IgE in their sera than HCs $(14,16)$. In line with these previous findings, the present study showed significantly higher levels of $\operatorname{IgE}$ in $\mathrm{CD}$ patients than in HCs. We also found that the levels of total serum IgE were positively correlated with food-specific IgGs against egg, milk, and wheat in CD patients. A likely explanation is that the immune system in the intestinal mucosal of $\mathrm{CD}$ patients tends to react more actively against egg, milk, and wheat antigens than other dietary antigens. Such hyperreactivity of the immune response may lead to IgE-mediated allergic diseases. The prevalence of three food antigens (egg, milk, wheat) in provoking food allergy in IBD resembles the presence of food allergy in non-IBD patients. Therefore, we suspect that egg, milk and wheat antigens might be important food allergens in CD patients.

No significant correlation was noted between foodspecific IgGs and CDAI in our study. This may be due to the fact that CD patients do not reflect a distinct pathophysiology, and various social and cultural factors heavily influence the severity and frequency of their symptoms. Consequently, symptoms may fail to be distinguished between different underlying pathophysiologies. SES-CD may be a more precise method than CDAI for assessing colonic mucosal lesions, which reflects the real state of disease. However, both of these methods are strongly subjective, and their scores do not represent the actual state of disease $(48,49)$. Our study also showed that the immune response induced 
Table 4. Degree of Food-specific IgGs for Individual CD Patient before and after IFX Treatment.

\begin{tabular}{|c|c|c|c|c|c|c|c|c|c|c|c|c|c|c|c|}
\hline \multicolumn{2}{|c|}{ CD patients } & \multirow{2}{*}{$\frac{\text { Beef }}{-}$} & \multirow{2}{*}{$\begin{array}{c}\text { Chicken } \\
-\end{array}$} & \multirow{2}{*}{$\begin{array}{c}\text { Codfish } \\
-\end{array}$} & \multirow{2}{*}{$\frac{\text { Corn }}{1+}$} & \multirow{2}{*}{$\begin{array}{c}\text { Crab } \\
-\end{array}$} & \multirow{2}{*}{$\frac{\text { Egg }}{-}$} & \multirow{2}{*}{$\begin{array}{c}\text { Mushroom } \\
-\end{array}$} & \multirow{2}{*}{$\begin{array}{c}\text { Milk } \\
-\end{array}$} & \multirow{2}{*}{$\begin{array}{c}\text { Pork } \\
-\end{array}$} & Rice & Shrimp & Soybean & Tomato & Wheat \\
\hline 1 & B & & & & & & & & & & - & - & - & $2+$ & - \\
\hline & A & - & - & - & $1+$ & - & - & - & - & - & $1+$ & - & - & - & - \\
\hline 2 & B & - & - & $1+$ & $2+$ & - & $2+$ & - & - & - & $1+$ & - & - & $2+$ & - \\
\hline & A & - & - & - & - & - & $1+$ & - & - & - & - & - & - & - & - \\
\hline 3 & B & - & - & - & $2+$ & - & $2+$ & - & $1+$ & - & $1+$ & - & - & $1+$ & - \\
\hline & A & - & - & - & $1+$ & - & $2+$ & - & $2+$ & - & $1+$ & - & - & - & - \\
\hline 4 & B & - & - & - & - & - & $2+$ & - & $3+$ & - & - & - & - & - & - \\
\hline & A & - & - & - & - & - & - & - & $2+$ & - & - & - & - & - & - \\
\hline 5 & B & - & - & - & $2+$ & - & - & - & - & - & $1+$ & - & - & $2+$ & - \\
\hline & A & - & - & - & $2+$ & - & - & - & $1+$ & - & - & - & - & - & - \\
\hline 6 & $\mathrm{~B}$ & - & - & - & - & - & $3+$ & - & - & - & - & - & - & - & $2+$ \\
\hline & A & - & - & - & - & - & $2+$ & - & - & - & - & - & - & - & $1+$ \\
\hline 7 & B & - & - & - & - & - & - & - & - & - & - & - & - & $1+$ & - \\
\hline & A & - & - & - & - & - & - & - & - & - & - & - & - & - & - \\
\hline 8 & B & - & - & $1+$ & - & - & - & - & - & - & - & - & - & - & - \\
\hline & A & - & - & - & - & - & - & - & - & - & - & - & - & - & - \\
\hline 9 & B & - & - & - & - & - & $2+$ & - & - & - & - & - & - & - & - \\
\hline & A & - & - & - & - & - & - & - & - & - & - & - & - & - & - \\
\hline 10 & B & - & - & - & $3+$ & - & $3+$ & - & $3+$ & - & $3+$ & - & $3+$ & $1+$ & - \\
\hline & A & - & - & - & $3+$ & - & $3+$ & - & $3+$ & - & $3+$ & - & $3+$ & - & - \\
\hline 11 & B & - & - & $1+$ & $2+$ & - & $2+$ & - & - & - & $2+$ & - & $2+$ & $1+$ & - \\
\hline & A & - & - & - & - & - & - & - & - & - & - & - & - & - & - \\
\hline 12 & B & - & - & - & - & - & $3+$ & - & - & - & - & - & - & - & - \\
\hline & A & - & - & - & - & - & - & - & - & - & - & - & - & - & - \\
\hline 13 & B & - & - & - & - & - & $3+$ & - & $3+$ & - & - & - & $1+$ & $1+$ & - \\
\hline & A & - & - & - & - & - & $3+$ & - & $3+$ & - & - & - & - & - & $1+$ \\
\hline 14 & B & - & - & - & - & - & $1+$ & $2+$ & $3+$ & - & - & - & $1+$ & - & $1+$ \\
\hline & A & - & - & - & - & - & $1+$ & $1+$ & $3+$ & - & - & - & $1+$ & $1+$ & - \\
\hline 15 & B & - & - & - & - & - & $3+$ & - & $3+$ & - & - & $2+$ & $3+$ & - & $1+$ \\
\hline & A & - & - & - & $1+$ & - & - & - & - & - & $1+$ & - & $1+$ & $2+$ & $1+$ \\
\hline 16 & B & - & - & - & $2+$ & - & $2+$ & $1+$ & $1+$ & - & $2+$ & - & $1+$ & $2+$ & $1+$ \\
\hline & A & - & - & - & $2+$ & - & $1+$ & - & $2+$ & - & $2+$ & - & $1+$ & $1+$ & - \\
\hline 17 & B & - & - & - & $1+$ & - & - & - & - & - & $1+$ & - & - & $2+$ & - \\
\hline & A & - & - & - & - & - & - & - & - & - & - & $1+$ & - & - & - \\
\hline 18 & B & - & - & - & $3+$ & $1+$ & $3+$ & - & - & - & $2+$ & - & $1+$ & $3+$ & - \\
\hline & A & - & - & - & $2+$ & $1+$ & $3+$ & - & - & - & $1+$ & - & - & $2+$ & - \\
\hline 19 & B & - & - & - & - & - & $3+$ & - & - & - & - & - & - & $2+$ & $1+$ \\
\hline & A & - & - & - & - & - & - & - & - & - & - & - & - & - & - \\
\hline 20 & B & - & - & $3+$ & $2+$ & - & $3+$ & - & - & - & - & - & $3+$ & $1+$ & - \\
\hline & A & - & - & $1+$ & $1+$ & - & $2+$ & - & - & - & - & - & $1+$ & $1+$ & $1+$ \\
\hline 21 & B & - & - & - & - & - & $3+$ & - & $3+$ & - & $2+$ & - & $2+$ & - & $3+$ \\
\hline & A & - & - & - & - & - & $1+$ & - & $2+$ & - & $1+$ & - & - & - & $1+$ \\
\hline 22 & B & - & - & - & $2+$ & - & $3+$ & - & $1+$ & - & $2+$ & - & - & $3+$ & $3+$ \\
\hline & A & - & - & - & $2+$ & - & $3+$ & - & - & - & $2+$ & - & - & $2+$ & $3+$ \\
\hline 23 & B & - & - & - & - & $2+$ & - & - & - & - & - & - & - & - & - \\
\hline & A & - & - & - & - & $1+$ & - & - & - & - & - & - & - & - & - \\
\hline 24 & B & - & - & - & $2+$ & - & $2+$ & - & $3+$ & - & $2+$ & - & $2+$ & $3+$ & $2+$ \\
\hline & A & - & - & - & $2+$ & - & $1+$ & - & $3+$ & - & $2+$ & - & $1+$ & $3+$ & $1+$ \\
\hline 25 & B & - & - & - & $3+$ & - & $1+$ & - & $3+$ & - & $2+$ & - & $1+$ & $3+$ & $3+$ \\
\hline & A & - & - & - & $2+$ & - & $1+$ & - & $2+$ & - & $2+$ & - & $1+$ & $3+$ & $2+$ \\
\hline 26 & B & - & - & - & $1+$ & - & $2+$ & - & - & - & $2+$ & - & - & - & - \\
\hline & A & - & - & - & - & - & - & - & - & - & - & - & - & $1+$ & - \\
\hline 27 & B & - & - & $2+$ & $3+$ & - & $3+$ & - & $3+$ & - & $3+$ & - & $3+$ & $3+$ & $3+$ \\
\hline & A & - & - & $2+$ & $3+$ & - & $2+$ & - & $2+$ & - & $3+$ & - & $2+$ & $3+$ & $2+$ \\
\hline 28 & B & - & - & - & - & - & $3+$ & - & - & - & - & - & - & - & - \\
\hline & A & - & - & - & - & - & $1+$ & - & - & - & - & - & - & - & - \\
\hline 29 & B & - & - & - & - & - & $1+$ & - & - & - & - & - & - & - & - \\
\hline & A & - & - & - & - & - & - & - & - & - & - & - & - & - & - \\
\hline 30 & B & - & - & - & - & - & $3+$ & $2+$ & $2+$ & - & - & - & - & - & - \\
\hline & A & - & - & - & $1+$ & - & $3+$ & $1+$ & $1+$ & - & $1+$ & - & - & $2+$ & - \\
\hline 31 & B & - & - & - & $3+$ & - & $3+$ & - & - & - & $3+$ & - & $3+$ & $2+$ & - \\
\hline & A & - & - & - & $1+$ & - & $3+$ & - & - & - & $3+$ & - & $2+$ & $1+$ & - \\
\hline
\end{tabular}

CD: Crohn's disease, IFX: infliximab, B: before IFX treatment, A: after IFX treatment 
A
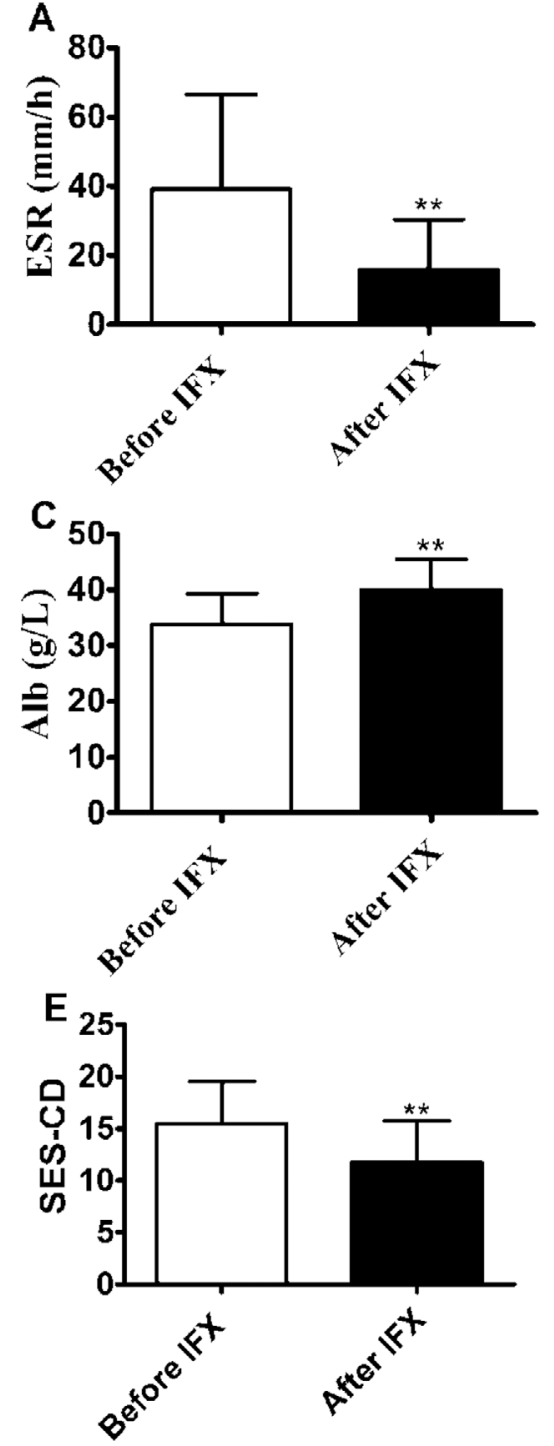

B
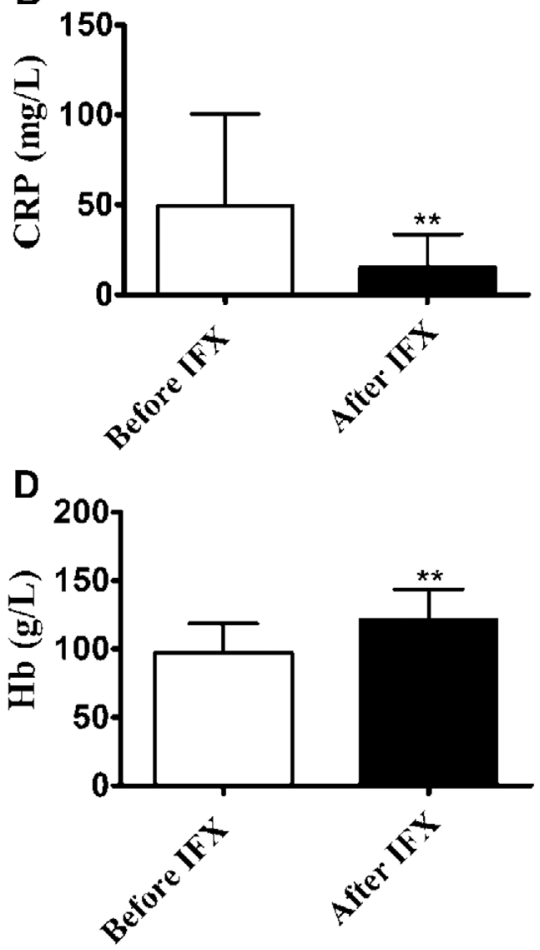

Figure 5. Infliximab (IFX) therapy induces clinical remission and promotes intestinal mucosal healing in CD patients. The levels of ESR (A), CRP (B), Alb (C), Hb (D), and SES-CD (E) were determined in IFX-treated patients with active CD before and after IFX treatment, as described in the Materials and Methods. **p<0.01 compared with those before IFX treatment. CD: Crohn's disease, ESR: erythrocyte sedimentation rate, CRP: C-reactive protein, Alb: albumin, Hb: hemoglobin, SESCD: simple endoscopic score for CD

by dietary antigens was independent of sex, disease location, and disease behavior, and age was found to correlate with food-specific IgGs.

In the present study, IFX treatment was able to downregulate the immune responses induced by dietary antigens. Thus far, no decisive evidence supporting the efficacy of IFX to reduce food allergies has been obtained. However, it was reported in a previous study that the levels of antimicrobial antibodies in $\mathrm{CD}$ patients receiving anti-TNF treatment were lower than those in $\mathrm{CD}$ patients that did not receive anti-TNF treatment (50). In the present study, we observed decreased levels of food specific-IgGs after IFX administration. We hypothesized that food antigens and microbial antigens were all environmental antigens. Therefore, we inferred that IFX treatment might alleviate food intoler- ance induced by food specific-IgGs. If anti-TNF treatment affects the levels of food specific-IgGs, it is unlikely to be due solely to the effects of this treatment on dampening active disease and acute inflammation, since the levels of food specific-IgGs are independent of clinical disease activity. However, anti-TNF therapy is the most effective approach for the induction of long-term remission and mucosal healing. It is conceivable that suppression of intestinal inflammation and repair of intestinal barrier defects may lead to decreased food induced immune responses $(29,51)$, Furthermore, the long-term inhibition of intestinal inflammation and direct targeting of memory $\mathrm{T}$ and $\mathrm{B}$ lymphocytes by IFX may also lead to a reduction in the levels of food-specific IgGs $(52,53)$. Therefore, we should interpret these findings with caution. More samples will be included in our future 
study to confirm the current hypothesis. Due to the rich diversity of food and non-food allergens in the gut lumen, the immune reactions can be very distinct for different allergens, and the mechanisms underlying how food specific- $\operatorname{IgG}$ functions after IFX treatment may be complicated, meriting further study.

A similar investigation to the present study was performed previously (35). However, conspicuous differences exist between that study and our own. In the previous study, the authors first identified the positive state against 14 common food allergens for every CD and UC patient as well as HC. Then, based on these positive subjects, the positive rates of food-specific $\mathrm{IgG}$ antibodies against 14 common food allergens were determined. In this way, the authors found that the top five most-prevalent food allergens causing positive $\mathrm{IgG}$ antibodies in $\mathrm{CD}$ patients were egg, rice, corn, tomato, and soybean. However, with this data processing method, the authors could not tell if there were significant differences in the 14 common food allergens among the $\mathrm{CD}$, UC, and HC groups. Our study's sample size (301 patients) was much larger than that in the preceding study (112 samples). With this enlarged sample size, we were able to perform comparisons among the $\mathrm{CD}, \mathrm{UC}$, and $\mathrm{HC}$ groups and assess the significance of differences among these groups from a statistical perspective, which has yielded more direct implications for clinical practice.

However, despite efforts to improve our study, limitations still exist. First, the IBD patients initially included in this study were on different medications, and these medical treatment certainly could have influenced the measured parameters. However, we kept the medications constant for the study period in order to minimize such bias. Second, we only tested 14 common food antigens in this study, which may have resulted in an insufficient assessment of food intolerance. Therefore, more foodstuffs should be included in future studies to identify sensitivities. Third, more patients who received IFX treatment should be recruited in the future, and the follow-up time should be sufficient for each patient.

In summary, this study has demonstrated that CD patients have elevated food-specific IgGs against common daily food antigens, such as egg, milk, wheat, corn, rice, tomato, codfish, and soybean. These food-specific IgGs may mediate similar pathophysiological routes in $\mathrm{CD}$ and other atopic diseases. Our study also revealed that egg, milk, and wheat allergy are more frequent in CD patients than other common daily foods, suggesting that optimizing CD dietary categories may be warranted. IFX treatment may inhibit the immune response induced by food antigens by suppressing intestinal inflammation and promoting mucosal healing. Therefore, food-specific IgGs may serve as an aid in the diagnosis and management of food allergy in cases of clinically active IBD.

The authors state that they have no Conflict of Interest (COI).
Nanping Xiao and Fenghua Liu contributed egually to this work.

\section{References}

1. Baumgart DC, Sandborn WJ. Crohn's disease. Lancet 380: 15901605, 2012.

2. Farrell RJ, Peppercorn MA. Ulcerative colitis. Lancet 359: 331340, 2002.

3. Zhou G, Yu L, Fang L, et al. CD $177^{+}$neutrophils as functionally activated neutrophils negatively regulate IBD. Gut 67: 1052-1103, 2018.

4. He C, Yu T, Shi Y, et al. MicroRNA 301A promotes intestinal inflammation and colitis-associated cancer development by inhibiting BTG1. Gastroenterology 152: 1434-1448, 2017.

5. Liu Z, Cao AT, Cong Y. Microbiota regulation of inflammatory bowel disease and colorectal cancer. Semin Cancer Biol 23: 543552, 2013.

6. Xavier R, Podolsky D. Unravelling the pathogenesis of inflammatory bowel disease. Nature 448: 427-434, 2007.

7. Zhou G, Liu Z. Potential roles of neutrophils in regulating intestinal mucosal inflammation of inflammatory bowel disease. J Dig Dis 18: 495-503, 2017.

8. Bernstein CN, Wajda A, Blanchard JF. The clustering of other chronic inflammatory diseases in inflammatory bowel disease: a population-based study. Gastroenterology 129: 827-836, 2005.

9. Myrelid P, Dufmats M, Lilja I, Grännö C, Lannerstad O, Sjödahl R. Atopic manifestations are more common in patients with Crohn disease than in the general population. Scand J Gastroenterol 39: 731-736, 2004.

10. Fireman E, Masarwy F, Groisman G, et al. Induced sputum eosinophilia in ulcerative colitis patients: the lung as a mirror image of intestine? Respir Med 103: 1025-1032, 2009.

11. Louis E, Louis R, Shute J, et al. Bronchial eosinophilic infiltration in Crohn's disease in the absence of pulmonary disease. Clin Exp Allergy 29: 660-666, 1999.

12. Walker MM, Powell N, Talley NJ. Atopy and the gastrointestinal tract-a review of a common association in unexplained gastrointestinal disease. Expert Rev Gastroentero Hepatol 8: 289-299, 2014.

13. Boeckxstaens G. Mast cells and inflammatory bowel disease. Curr Opini Pharmacol 25: 45-49, 2015.

14. Mekkel G, Barta Z, Ress Z, Gyimesi E, Sipka S, Zeher M. Increased IgE-type antibody response to food allergens in irritable bowel syndrome and inflammatory bowel diseases. Orv Hetil 146: 797-802, 2005 (in Hungarian, Abstract in English).

15. Crowe SE, Perdue MH. Gastrointestinal food hypersensitivity: basic mechanisms of pathophysiology. Gastroenterology 103: 10751095, 1992.

16. Levo Y, Shalit M, Wollner S, Fich A. Serum IgE levels in patients with inflammatory bowel disease. Ann Allergy 56: 85-87, 1986.

17. Brignola C, Miniero R, Campieri M, et al. Dietary allergy evaluated by PRIST and RAST in inflammatory bowel disease. Hepatogastroenterology 33: 128-130, 1986.

18. Huber A, Genser D, Spitzauer S, Scheiner O, Jensen-Jarolim E. $\operatorname{IgE} /$ anti-IgE immune complexes in sera from patients with Crohn's disease do not contain food-specific IgE. Int Arch Allergy Immunol 115: 67-72, 1997.

19. Bentz S, Hausmann M, Piberger $\mathrm{H}$, et al. Clinical relevance of $\mathrm{IgG}$ antibodies against food antigens in Crohn's disease: a doubleblind cross-over diet intervention study. Digestion 81: 252-264, 2010.

20. Awazuhara H, Kawai H, Maruchi N. Major allergens in soybean and clinical significance of IgG4 antibodies investigated by IgEand IgG4-immunoblotting with sera from soybean-sensitive patients. Clin Exp Allergy 27: 325-332, 1997. 
21. Høst A, Husby S, Gjesing B, Larsen J, Løwenstein H. Prospective estimation of $\mathrm{IgG}, \mathrm{IgG}$ subclass and $\mathrm{IgE}$ antibodies to dietary proteins in infants with cow milk allergy. Allergy 47: 218-229, 1992.

22. Dupaul-Chicoine J, Dagenais M, Saleh M. Crosstalk between the intestinal microbiota and the innate immune system in intestinal homeostasis and inflammatory bowel disease. Inflamm Bowel Dis 19: 2227-2237, 2013.

23. Satsangi J, Silverberg MS, Vermeire S, Colombel JF. The Montreal classification of inflammatory bowel disease: controversies, consensus, and implications. Gut 55: 749-753, 2006.

24. Best WR, Becktel JM, Singleton JW, Kern F. Development of a Crohn's disease activity index. Gastroenterology 70: 439-444, 1976.

25. Walmsley R, Ayres R, Pounder R, Allan R. A simple clinical colitis activity index. Gut 43: 29-32, 1998.

26. Daperno M, D'Haens G, Van Assche G, et al. Development and validation of a new, simplified endoscopic activity score for Crohn's disease: the SES-CD. Gastrointest Endosc 60: 505-512, 2004.

27. Dignass A, Van Assche G, Lindsay JO, et al. The second European evidence-based consensus on the diagnosis and management of Crohn's disease: current management. J Crohns Colitis 4: 2862, 2010.

28. Dignass A, Eliakim R, Magro F, Maaser C, Chowers Y, Geboes K, Mantzaris G, Reinisch W, Colombel J-F, Vermeire S. Second European evidence-based consensus on the diagnosis and management of ulcerative colitis part 1: definitions and diagnosis. J Crohns Colitis 6: 965-990, 2012.

29. Liu C, Xia X, Wu W, et al. Anti-tumour necrosis factor therapy enhances mucosal healing through down-regulation of interleukin21 expression and T helper type 17 cell infiltration in Crohn's disease. Clin Exp Immunol 173: 102-111, 2013.

30. Pabst O, Mowat AM. Oral tolerance to food protein. Mucosal Immunol 5: 232-239, 2012.

31. Van Den Bogaerde J, Cahill J, Emmanuel A, et al. Gut mucosal response to food antigens in Crohn's disease. Aliment Pharmacol Ther 16: 1903-1915, 2002.

32. Kawaguchi T, Mori M, Saito K, et al. Food antigen-induced immune responses in Crohn's disease patients and experimental colitis mice. J Gastroenterol 50: 394-405, 2015.

33. Nasu J, Mizuno M, Uesu $T$, et al. Increased incidence of allergic disorders and elevated food-specific serum IgG4 levels in Japanese patients with Crohn's disease. Allergol Int 48: 247-251, 1999.

34. Lerner A, Rossi TM, Park B, Albini B, Lebenthal E. Serum antibodies to cow's milk proteins in pediatric inflammatory bowel disease: Crohn's disease vs. ulcerative colitis. Acta Paediatr Scand 78: 81-86, 1989.

35. Cai C, Shen J, Zhao D, et al. Serological investigation of food specific immunoglobulin $\mathrm{G}$ antibodies in patients with inflammatory bowel diseases. PLoS One 9: e112154, 2014.

36. Hvatum M, Scott H, Brandtzaeg P. Serum IgG subclass antibodies to a variety of food antigens in patients with coeliac disease. Gut 33: 632-638, 1992.

37. Tay SS, Clark AT, Deighton J, King Y, Ewan PW. Patterns of immunoglobulin $\mathrm{G}$ responses to egg and peanut allergens are distinct: ovalbumin-specific immunoglobulin responses are ubiquitous, but peanut-specific immunoglobulin responses are upregulated in peanut allergy. Clin Exp Allergy 37: 1512-1518,
2007.

38. Shek LP, Bardina L, Castro R, Sampson HA, Beyer K. Humoral and cellular responses to cow milk proteins in patients with milkinduced IgE-mediated and non-IgE-mediated disorders. Allergy 60: 912-919, 2005

39. Jowett SL, Seal CJ, Pearce MS, et al. Influence of dietary factors on the clinical course of ulcerative colitis: a prospective cohort study. Gut 53: 1479-1484, 2004.

40. Zeissig S, Burgel N, Gunzel D, et al. Changes in expression and distribution of claudin 2, 5 and 8 lead to discontinuous tight junctions and barrier dysfunction in active Crohn's disease. Gut 56: 61-72, 2007.

41. Malbec O, Daëron M. The mast cell IgG receptors and their roles in tissue inflammation. Immunol Rev 217: 206-221, 2007.

42. Meulenbroek LA, de Jong RJ, den Hartog Jager CF, et al. IgG antibodies in food allergy influence allergen-antibody complex formation and binding to B cells: a role for complement receptors. J Immunol 191: 3526-3533, 2013.

43. Belut D, Moneret-Vautrin D, Nicolas J, Grilliat J. IgE levels in intestinal juice. Dig Dis Sci 25: 323-332, 1980.

44. Kolmannskog S, Haneberg B. Immunoglobulin E in feces from children with allergy. Int Arch Allergy Immunol 76: 133-137, 1985.

45. Marcucci F, Sensi L, Bizzarri G. Specific IgE to food and inhalant allergens in intestinal washings of children affected by atopic eczema. Clin Exp Allergy 15: 345-354, 1985.

46. Karlsson T, Ellerson J, Dahlbom I, Bennich H. Analysis of the serum IgE levels in nonimmunized rats of various strains by a radioimmunoassay. Scand J Immunol 9: 217-228, 1979.

47. Baird A, Cuthbert A, Pearce F. Immediate hypersensitivity reactions in epithelia from rats infected with Nippostrongylus brasiliensis. Br J Pharmacol 85: 787-795, 1985.

48. Sipponen T, Nuutinen H, Turunen U, Färkkilä M. Endoscopic evaluation of Crohn's disease activity: comparison of the CDEIS and the SES-CD. Inflamm Bowel Dis 16: 2131-2136, 2010.

49. Yeşil A, Senateş E, Bayoğlu IV, Erdem ED, Demirtunç R, Kurdaş Övünç AO. Red cell distribution width: a novel marker of activity in inflammatory bowel disease. Gut Liver 5: 460, 2011.

50. Frehn L, Jansen A, Bennek E, et al. Distinct patterns of IgG and IgA against food and microbial antigens in serum and feces of patients with inflammatory bowel diseases. PLoS One 9: e106750, 2014.

51. Yu L, Yang X, Xia L, et al. Infliximab preferentially induces clinical remission and mucosal healing in short course Crohn's disease with luminal lesions through balancing abnormal immune response in gut mucosa. Mediators Inflamm 2015: 793764, 2015.

52. Li Z, Vermeire S, Bullens D, et al. Anti-tumor necrosis factor therapy restores peripheral blood B-cell subsets and CD40 expression in inflammatory bowel diseases. Inflamm Bowel Dis 21: 27872796, 2015.

53. Timmermans WM, van Laar JA, van der Houwen TB, et al. B-cell dysregulation in Crohn's disease is partially restored with infliximab therapy. PLoS One 11: e0160103, 2016.

The Internal Medicine is an Open Access article distributed under the Creative Commons Attribution-NonCommercial-NoDerivatives 4.0 International License. To view the details of this license, please visit (https://creativecommons.org/licenses/ by-nc-nd/4.0/).

(C) 2018 The Japanese Society of Internal Medicine Intern Med 57: 2787-2798, 2018 